\title{
Phronesis
}

\section{Ficelles, bricolage ou processus de conceptualisation : à propos des pratiques des chercheurs en sciences humaines et sociales}

\author{
Catherine Gouédard, Mélissa Arneton, Line Numa-Bocage et Jean-Claude \\ Kalubi
}

Volume 9, numéro 3-4, 2020

Les ficelles de l'analyste

URI : https://id.erudit.org/iderudit/1073576ar

DOI : https://doi.org/10.7202/1073576ar

Aller au sommaire du numéro

Éditeur(s)

Université de Sherbrooke

Champ social éditions

ISSN

1925-4873 (numérique)

Découvrir la revue

Citer ce document

Gouédard, C., Arneton, M., Numa-Bocage, L. \& Kalubi, J.-C. (2020). Ficelles, bricolage ou processus de conceptualisation : à propos des pratiques des chercheurs en sciences humaines et sociales. Phronesis, 9(3-4), 1-10.

https://doi.org/10.7202/1073576ar d'utilisation que vous pouvez consulter en ligne. 


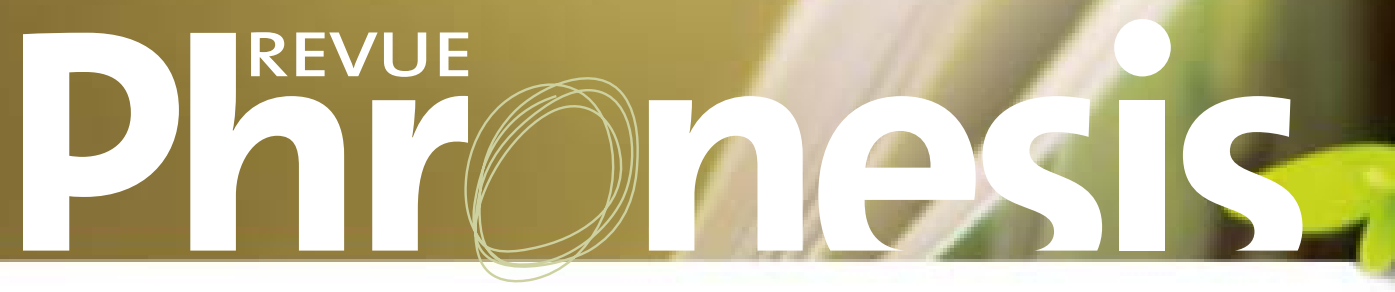

Ficelles, bricolage ou processus de conceptualisation: à propos des pratiques des chercheurs en sciences humaines et sociales

\author{
Introduction au numéro
}

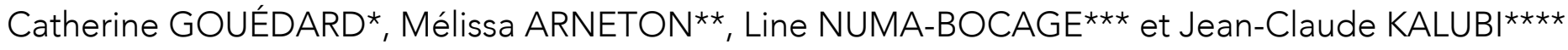

*Université Paris 8, Saint-Denis, UPL Université Paris Lumières, France

catherine.gouedard@univ-paris8.fr

${ }^{\star *}$ Institut national supérieur formation et recherche-handicap et enseignements adaptés (INSHEA), Suresnes, UPL Université Paris Lumières, France

melissa.arneton@inshea.fr

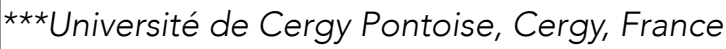

line.numa-bocage@cyu.fr

****Université de Sherbrooke, Sherbrooke, Canada

Jc.kalubi@usherbrooke.ca 
En parlant des défis de la globalisation dans les sociétés contemporaines, plusieurs auteurs stigmatisent de fortes pressions subies au sein des systèmes de formation pour promouvoir des normes de performance et d'efficacité. Le recours aux modèles d'efficacité s'accompagne de cadres d'action permettant de mieux analyser les politiques et pratiques éducatives.

Or, l'éducation constitue l'un des principaux terrains où se chevauchent les efforts d'adaptation de la personne et les tentatives collectives de réponses progressives. Elle offre une tendance quelque peu énigmatique pour I'analyste. Elle exige de ce dernier un renouvellement de ses réflexions vis-à-vis des problèmes rapportés, une attention aux impératifs de la réorganisation des services, ainsi qu'une affirmation de son rôle spécifique par rapport à la production de sens. Les effets produits au cours de l'agir réflexif de l'analyste renforcent les pratiques responsables, en vue d'améliorer l'efficacité des pratiques et de renouveler les approches. Dans les pages ci-après seront abordées tour à tour la métaphore de la « ficelle » et ses prolongements possibles au niveau conceptuel, le lien entre explicitation de l'activité et médiation, les enjeux d'analyser les expériences signifiantes de chercheur. Un regard transversal sur les contributions de ce numéro invite à poursuivre les investigations pour saisir les formes d'organisation complexes de l'activité des chercheurs.

\section{La métaphore de la « ficelle »}

Pour aborder les situations complexes au sein desquelles les phénomènes surgissent, les chercheurs recourent à de multiples principes épistémologiques et diverses stratégies spécifiques, correspondant à ce que Becker (2002) appelle une "ficelle »: " ces ficelles [...] ont pour fonction de suggérer de nouvelles manières de manipuler les choses, de permettre d'observer les choses sous un angle différent, afin de faire progresser la recherche en suscitant de nouvelles questions, de nouvelles possibilités de comparaison, d'invention de nouvelles catégories, etc. » (p. 29-30). Cette métaphore aide à aborder, à travers un vocabulaire apparemment simple, de nombreuses démarches empiriques de résolution de problèmes qui se présentent à différents moments de la recherche. Elle évoque tout d'abord l'idée d'un " truc simple » qui aide le chercheur à résoudre les problèmes méthodologiques et théoriques relevant de son projet spécifique. Un regard sur les pratiques de recherche au quotidien montre que certains chercheurs peuvent sembler « cachottiers » quant aux principes d'action guidant leurs tâches d'analyse, notamment ceux en sciences humaines et sociales (SHS). En fait, il n'est pas si simple de rendre compte de l'inventivité dans sa propre activité de chercheur et de la manière dont elle s'organise. Ainsi, une part importante de la créativité des chercheurs échappe à l'attention même de leurs collaboratrices et collaborateurs immédiats. Si l'explication de la négociation de l'objet, étudiée dans le cadre de recherches participatives ou concernant l'accès aux terrains notamment, fait l'objet de publications scientifiques (Desgagné, 1997; Morrissette, Pagoni et Pépin, 2017), les problèmes spécifiques suscités par les tâches d'analyse ne sont que partiellement exposés. Élucider comment le chercheur procède et comment des connaissances en relation avec ce processus s'élaborent, reste donc encore un défi. Ficelles (Becker, 2002) ou encore bricolage (Crettaz, 1986), ces métaphores circulent chez de nombreux professionnels pour évoquer des tours de main, des manières de faire, des procédés spécifiques qui sont difficilement dicibles. Car, ils sont d'une part, incorporées dans les activités et situations de travail; et d'autre part, ils sont en lien avec l'expérience de chacun. La ficelle aurait un statut méthodologique. Loin d'être figée, elle serait ainsi vivante. 
Toutefois, ces métaphores, aussi intéressantes soient-elles, peuvent poser des limites à la description même de ce qu'elles recèlent, et donc à leur compréhension et leur appropriation par un tiers. Ainsi, passer de la métaphore, qu'il s'agisse de ficelles ou de bricolage, au concept de conceptualisation conduit à aller plus loin dans la mise en visibilité de ce qui constitue une ficelle et à quoi renvoie ce bricolage. Les schèmes ${ }^{1}$, organisations invariantes de I'activité pour une classe donnée de situations, sont souvent implicites et demandent à être explicités (Vergnaud, 1985, 1996).

Questionner l'activité, en la décrivant et en l'analysant en profondeur, peut alors être un des moyens pour lever le voile sur ce que sont et à quoi servent les ficelles élaborées consciemment ou intuitivement par les chercheurs et comment elles sont mises en œuvre. Cette démarche peut contribuer à expliciter les conceptualisations qui sous-tendent les compétences du chercheur lorsqu'il doit résoudre des difficultés et faire face à des inquiétudes particulières survenant dans le parcours de recherche. En effet, quelques questions sont transmises d'une génération à l'autre: comment se fait et se conduit une recherche? Comment se constituent les dispositifs destinés à récolter des données? Tout dispositif d'analyse est-il suffisant pour favoriser l'accès à un phénomène spécifique? Comment choisir un dispositif ou le créer lors du processus complexe de la recherche en relation avec le(s) terrain(s) concerné(s)? Comment s'élabore, chemin faisant, une vision circulaire, notamment lorsque le chercheur fait corps avec ses données? Comment le chercheur compose-t-il avec l'incertitude et quelle place laisser à l'inattendu? Comment s'y prend un tel, comment s'y prennent-ils tous pour agir avec compétence? La plupart de ces questions font écho à des réflexions soulevées depuis plus de deux décennies dans des contextes professionnels variés.

Qu'il s'agisse de l'analyse d'activités d'enseignants (Rogalski, 2004; Goigoux, 2007; Clanet et Talbot, 2012), de celles de soignants (Belpaume, 2009), d'architectes (Raynaud, 1999) ou encore d'opérateurs de conduite dans le secteur industriel (Pastré, 1999), l'identification de ce qui a été fait, de ce que les professionnels disent de ce qu'ils ont fait, mais aussi de ce qui aurait pu être fait, contribue à comprendre leurs fonctionnements dans des situations familières, mais aussi comment ils s'adaptent à des situations inédites (Clot, Faïta, Fernandez et Scheller, 2000; Rix-Lièvre, 2010). Selon Rabardel (2005), le sujet s'appuie non seulement sur des ressources, internes et externes, pour mener à bien son activité, mais aussi en développe de nouvelles au cours de son activité. Enfin, dans une approche instrumentale, la ficelle peut s'entendre comme une ressource, comme peut l'être l'établi du bricoleur avec ses différentes composantes. Dans un processus d'appropriation et de développement, cette ficelle participerait à l'enrichissement et à la réorganisation des schèmes du chercheur, transformant alors ses rapports à l'objet de l'activité, aux autres et à lui-même.

\section{L'explicitation de l'activité du chercheur et sa relation avec la médiation}

La question de comment les compétences se forment en situations renvoie tout particulièrement à l'objet de la didactique professionnelle (Pastré, 2002; Pastré, Mayen et Vergnaud, 2006). Expliciter comment des savoirs se construisent en situation invite à considérer la place essentielle des classes de situation et des schèmes dans la construction des connaissances des professionnels. Le terme de didactique ne se centre pas, dans le cas présent, sur comment enseigner les savoirs et contenus d'une discipline, mais bien, sur ce qui guide et fait l'activité professionnelle, et plus précisément, dans le cas étudié, celle relative au chercheur.

Dans le cadre de l'analyse de l'activité scientifique, le courant pratique en épistémologie des sciences a contribué à montrer comment se construit et s'exprime la professionnalité de chercheurs en sciences et techniques ou en mathématiques par exemple, à partir de l'étude minutieuse en situation de comment ils construisent leur objet à partir de quels gestes et avec quels outils et réglages (Hacking, 2005; Soler \& Zwart, 2013). Ces travaux ont permis de mettre en lumière la dimension sociale de la science qu'il s'agisse de la définition de ce qui est scientifique (Daoust, 2018), des négociations des objets à investiguer (Jouvenet, 2007 ; Latour, 2005) ou de comment communiquer la science (Boch et Rinck, 2010).

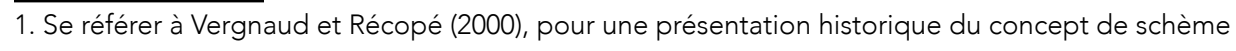


La question de la construction de l'identité de chercheur en tant qu'acteur inscrit dans une communauté de pratiques ayant des normes spécifiques partagées a également été investiguée, notamment dans une perspective sociologique (Gingras, 1991; Hackett, 2005). Toutefois, ces objets de questionnement n'ont pas été approfondis par des analyses centrées sur l'organisation de l'activité du chercheur, qu'elle se déroule en individuel ou avec d'autres. Des investigations ancrées dans les activités concrètes et singulières du chercheur restent rares, et ce, tout particulièrement en sciences humaines et sociales (Allamel-Raffin, Dupouy et Gangloff, 2019).

En explorant les travaux sur le sujet, les contributions portent notamment sur les liens entre le chercheur, le terrain ou l'objet de sa recherche dans une tradition abordant la question de l'engagement en recherche ainsi que la nature de la contribution du chercheur aux données produites (Spire, 1999; Becker, 2002; Gremion, 2016). Dans ce cadre, l'éthique, en tant que cadre normatif, est interrogée, mais, au-delà des discours, il reste à étudier, comment dans la pratique quotidienne de recherche, elle est mise en œuvre en regard d'autres schèmes avec lesquels elle peut être en concurrence ou se poser en termes de complémentarité (Capdevielle-Mougnibas et de Léonardis, 2010; Fassin, 2008; Larouche, 2019; Van Campenhoudt, 2000). L'essor de l'Open Science et de dispositifs d'aide à l'élaboration de protocoles ne doit ainsi pas occulter l'intérêt d'investiguer les pratiques des chercheurs eux-mêmes et le cadre institutionnel dans lequel ils conçoivent, mènent, conduisent des travaux scientifiques. Si l'intérêt renouvelé pour des démarches participatives en sciences humaines et sociales amène, en particulier, à se focaliser sur la manière dont différents acteurs contribuent à l'élaboration d'un projet de recherche (Niewiadomski, Portelance et Perez-Roux, 2017; Deschenaux, 2013), il convient également d'interroger la professionnalité propre du chercheur dans le quotidien de sa pratique en tant que pierre angulaire d'une démarche de recherche qu'elle soit ou non dans un cadre collaboratif. En effet, y compris lorsque tous les acteurs participent à l'analyse des données co-produites, le chercheur contribue par ses pratiques professionnelles à rendre intelligibles et partagées les données obtenues par l'ensemble des acteurs. Il contribue, ainsi à ce que chacun s'en saisisse, pour dépasser ou changer ce qu'une analyse d'un acteur singulier aurait pu produire comme connaissance (Numa-Bocage, 2014; Fossion, Jamin et Faulx, 2018).

Reprises dans les débats actuels sur la place et les missions des scientifiques, de telles analyses relancent l'intérêt d'une réflexion approfondie sur les pratiques méthodologiques des chercheurs en sciences humaines et sociales, leurs expériences d'analyse des données ainsi que la mise en œuvre de nouvelles stratégies de traitement les concernant. Vinck (2009) évoque ainsi l'écrit, entre autres, en tant « objet intermédiaire » dans la structuration des activités de recherches et la constitution d'un espace de savoirs. Il le théorise en tant que " médiateur », pour chacun et pour tous, aussi bien dans les réseaux de coopération scientifique que dans des processus de conception plus individuels. Le passage par l'écriture et la formalisation dans un format scientifique, de ce que sont les ficelles pour chacun des chercheurs s'engageant dans une démarche d'explicitation, s'inscrirait alors dans un processus de conceptualisation impliquant diverses médiations.

\section{Les expériences signifiantes de chercheur}

S'interroger sur l'activité des chercheurs en sciences humaines et sociales suppose de saisir des conceptualisations sous-jacentes dans l'interrelation entre défis méthodologiques, corpus spécifiques et expériences originales vécues dans leur quotidien scientifique. Deux colloques internationaux, consacrés aux ficelles de l'analyste, initiés et montés par l'association pour la recherche sur le développement des compétences (ARDéCO), ont visé à créer des espaces d'échanges pour les chercheurs sur leurs pratiques. Un premier symposium fermé "Les ficelles de I'analyste » organisé par Line Numa-Bocage et Jean-Claude Kalubi s'est tenu le 8 juin 2016 à l'occasion du colloque OPÉEN \& REFORM «Observer pour former » dans le cadre des journées scientifiques de l'Université de Nantes en France. Les premiers échanges tenus dans ce groupe de travail, réunissant chercheurs et étudiants de cycles supérieurs en provenance de différents pays, ont suscité l'intérêt de faire grandir la réflexion sur les démarches de recherche. Ce symposium a conduit à l'organisation d'un colloque international, qui s'est tenu les 3 et 4 juillet 2017 à I'Université Paris-Descartes en France. 
Ce colloque organisé par l'association ARDéCo a été monté en collaboration avec différents laboratoires et universités au Canada, en Italie et en France ${ }^{2}$. Chacun des participants (chercheurs confirmés et doctorants) était invité à présenter, à partir de ses expériences signifiantes, des questions soulevées dans sa pratique et les réponses qu'il y a apportées en qualité d'analyste afin d'enrichir la réflexion sur l'inventivité dans la recherche en SHS. Inviter les chercheurs à mener une analyse réflexive sur leur activité est une des manières d'identifier ce que sont, et à quoi servent les ficelles élaborées par les chercheurs et comment ils les mettent en œuvre. Que les ficelles interviennent et se développent lors de la construction d'un projet de recherche, lors des questionnements en rapport avec les théories, questions et objets de la recherche, dans le processus des mises en récits ou de l'écriture de la recherche, l'enjeu était d'interroger ce qui accompagne la créativité du chercheur, ce qui soutient le développement et la production des savoirs au cours de la recherche en SHS, et ce qui contribue à construire l'identité même du chercheur. L'enjeu était aussi d'ouvrir des perspectives d'application en vue de soutenir les démarches concrètes en faveur de I'amélioration des projets à venir et, en particulier, la formation à la recherche des étudiants (doctorants ou « masterants »). Le passage à l'écrit est une occasion pour le chercheur, qu'il s'inscrive en psychologie, en sciences de l'éducation ou en sociologie, de se pencher sur son activité, d'y revenir à distance, et d'en cerner certains phénomènes marquants. Le partage des ficelles, ouvert à l'interdisciplinarité, peut contribuer à soutenir des démarches concrètes en faveur de l'avancée de projets d'autres chercheurs, à la mise en œuvre d'une démarche d'analyse ou plus généralement à la formation à la recherche de doctorants, futurs professionnels de la recherche. Considérant les différentes disciplines comme ayant des points communs entre elles, en sus de différences, ce partage peut aussi contribuer à dégager des invariants au-delà des spécificités.

\section{La présentation des contributions}

Le présent numéro «Expliciter les ficelles de l'analyste » regroupe différentes contributions éclairant la thématique selon différents éclairages. II s'agit de contributions originales utiles à la compréhension du problème des ponts entre la théorie et la pratique de l'analyste. S'appuyant notamment sur des projets en cours ou réalisés, chaque chercheur ou collectif de travail s'attache à présenter les réponses apportées en qualité d'analyste aux questions qu'il se pose et aux nouvelles questions qui émergent. Chaque contribution part des expériences propres du chercheur, pour enrichir la réflexion et dégager des enseignements pertinents pour l'explicitation des pratiques de recherche.

Dans le premier article intitulé Représentations du pouvoir communicationnel des parents: synthèse sur deux décennies d'analyse réflexive en partenariat, Jean-Claude Kalubi et Rosly Angrand, s'attachent à expliciter les ficelles de l'analyste pour construire la dynamique de l'empowerment et du pouvoir communicationnel des participants qui interagissent dans des groupes de réflexion selon une perspective écosystémique. En partant de la compréhension des ajustements constatés, des processus de résolution de problèmes, ainsi que de l'évolution des représentations individuelles et collectives, ces ficelles sont déclinées selon différentes catégories.

Le deuxième article, s'inscrivant également dans une démarche réflexive collective, se focalise plus spécifiquement sur Les ficelles de la collaboration dans l'analyse de l'activité au sein d'un groupe de recherche collaborative. Ses auteurs, Bruno Hubert, Christine Pierrisnard et Marie-Paule Vannier, posent d'emblée «la ficelle » comme relevant d'un processus de conceptualisation au cours de l'activité afin de répondre aux exigences de la démarche de recherche scientifique. L'activité étudiée se déroule dans un groupe de recherche collaborative réunissant cinq formateurs en institut de formation de soins infirmiers et trois chercheurs en sciences de l'éducation, dont l'enjeu est de proposer un accompagnement en formation qui s'appuie sur l'analyse de récits. Les auteurs, à partir d'une analyse des échanges enregistrés en séances de groupe, interrogent les conditions de cette collaboration et, en particulier, la place du récit et de son analyse comme constituant une « ficelle » pour favoriser la démarche réflexive sur les pratiques.

2. Pour plus d'informations sur le colloque, consulter le site web de l'association http://www.ardeco-association.fr/colloque-les-ficelles-lanalyste/ 
Line Numa-Bocage présente une méthode, fondée sur les concepts de la didactique professionnelle (Pastré, 2002), et utilisée régulièrement dans ses recherches collaboratives pour comprendre l'activité des professionnels: I'entretien d'analyse de l'activité en didactique professionnelle: I'EA-CDP (entretien en auto-confrontation à visée de didactique professionnelle). Elle s'attache à présenter les caractéristiques récurrentes de cette forme d'entretien qu'elle mène, dans différents contextes, à partir de deux exemples d'entretiens menés avec des professionnels dans le secteur de la santé. Ces caractéristiques relèveraient donc de sa ficelle de chercheuse. Ficelle "qui suggère une certaine manière d'observer, de questionner, de conceptualiser, de raisonner », et qui se révèle être un instrument psychologique guidant les interventions d'analyste pendant l'entretien. Ce souci d'explicitation s'inscrit dans la préoccupation de partager une méthode avec des doctorants et d'autres chercheurs, et de l'intégrer en formation professionnelle.

Naïr Tuboiti, Line Numa-Bocage, Lêda Freitas développent une proposition méthodologique, à partir de recherches menées au Brésil, pour accompagner le développement des professionnels qui interviennent dans les classes publiques d'alphabétisation. La ficelle des chercheuses est de reprendre, pour la formation même des enseignantes, les principes de l'approche du post-constructivisme (Grossi, 2005) fondés sur l'apprentissage comme phénomène social. Dans leur article Groupes auliques et Groupe d'études : procédure du post-constructivisme d'enseignement et apprentissage, elles présentent en quoi la formation-en-action, la liaison avec une communauté scientifique (le GEEMPA, Groupe d'Études sur l'Éducation, la Méthodologie de la Recherche et l'Action), la participation hebdomadaire de ces enseignantes au groupe d'études, sont des facteurs favorisant la compréhension en retour des processus d'apprentissage chez les élèves.

L'article de Tommy Collin-Vallée et Maryvonne Merri, se centre sur l'expérience du chercheur doctorant. Dans leur article, Un corpus nommé désir: le laboratoire d'étonnement pour réintroduire l'affect dans la recherche. De l'individuel au collectif, la ficelle méthodologique qu'ils explicitent se déploie dans un "laboratoire d'étonnement ». S'appuyant sur le propre vécu d'un chercheur (le premier auteur de l'article), ils soulèvent l'importance d'une méthodologie permettant au chercheur de multiplier ses points de vue sur un corpus, et de confronter ses interprétations aux autres dans ce laboratoire, afin que l'angoisse puisse devenir source d'étonnement, et donc environnement collectif capacitant pour le chercheur. Ils soulignent qu'il est essentiel de considérer les dimensions émotionnelles, qui ont encore insuffisamment de place à l'Université, dans la construction de la recherche.

Marie Sophie Madiba et Nadja Maria Acioly-Regnier dans leur article Construction du sens et de la signification des images de campagnes présidentielles de 2007 et 2012 dans la presse française soulèvent l'importance d'une analyse à partir des propriétés de l'objet d'étude lui-même, pour que le chercheur puisse laisser davantage de place à la découverte. Ces auteures, à partir des ficelles « de la relation et de la différence », qui sont complémentaires, montrent comment le sens et la signification émanent du discours des journaux sur les campagnes présidentielles. La «ficelle de la relation » s'appuie en particulier sur le postulat latourien suivant: c'est en questionnant les relations entre les différentes composantes matérielles et humaines d'une activité que le sens peut émerger à l'analyste (Latour, 2007). En recourant à la «ficelle de la différence », le chercheur réserve de même une place au doute, à la découverte, à la singularité pour affiner son interprétation, dans l'analyse.

En immergeant le lecteur dans son cheminement et son processus de transformation sur une longue temporalité, celle de la réalisation d'une thèse de doctorat, François Gremion témoigne de l'importance du récit à soi-même dans la transformation du « soi » professionnel et personnel. Son article, L'évolution d'une posture de recherche: le soutien par l'analyse en mode écriture est un récit autobiographique. Il décrit avec minutie sa démarche d'analyse en mode écriture (Paillé et Mucchielli, 2003), et comment cette méthode, qui est pour lui sa principale ficelle d'analyste, a transformé le rapport à l'objet de recherche, aux situations et aux acteurs. Le concept ou l'objet de recherche désormais s'apparentent à un personnage de roman: "Quelle histoire me raconte-t-il? ; " la vision du monde où tout est donné d'avance, avec ses lois et sa finalité » devient " un monde en construction et à construire »; la conception du sujet et de son développement se transforme aussi au travers de ce processus. II montre également comment cette méthode a reconfiguré des dimensions personnelles, dont son identité de chercheur. 
Mélissa Arnéton et Séverine Mayol questionnent la formation à la recherche, en prenant appui sur une expérience de formation auprès d'étudiants en Master, dans leur article intitulé Former des analystes en sciences sociales. Considérer les ficelles de la méthode comme outils d'enseignement. Elles soulèvent la complexité de cette mission. Elles présentent une démarche d'enquête collective tutorée visant à développer des schèmes du chercheur pour les étudiants en formation. Procédant par déconstruction et construction d'une démarche de recherche dans un dispositif d'inspiration socioconstructiviste, le cadre d'enseignement mis en place vise à inciter les étudiants à une démarche réflexive sur les méthodes et leurs articulations pour qu'ils deviennent acteurs et producteurs de connaissances relativement à leur objet de recherche et dépassent une vision techniciste de la méthodologie. Comme les auteures le disent, « le travail d'énonciation de la démarche de recherche est un travail difficile y compris pour les chercheurs expérimentés ». C'est donc aussi, pour elles, une occasion, à partir d'une expérience d'enseignement menée en duo, d'expliciter leurs ficelles d'analyste.

L'article de Sylviane Veillette et de Maryvonne Merri, À la recherche du monde vécu: Une alternative aux « données probantes » en psychologie de l'éducation développe, quant à lui, une vision critique sur l'approche dominante de la psychologie, étendue aujourd'hui à la psychologie de l'éducation. Ces auteures contrastent ainsi l'approche dominante d'une psychologie basée sur les « données probantes » avec l'approche minoritaire d'une "psychologie de l'éducation et du développement » (perspective vygotskienne) autour de trois concepts: celui de " participant » à la recherche, celui d' « objets d'étude » et celui d' " activité du chercheur ». Elles soulèvent en particulier l'importance aujourd'hui que la psychologie de l'éducation ne soit « plus dédiée à l'émission de lois mais plutôt à la qualification des phénomènes ». Du point de vue de la formation, elles montrent le défi, pour le développement scientifique, de former des étudiants à prendre en compte la complexité du réel et qu'ils deviennent enfin créateurs de méthodes.

Le lecteur remarquera que l'organisation des textes, ne suit pas la temporalité d'un projet dans ses étapes classiques de description: du démarrage d'un projet, en passant par la conception du protocole à l'interprétation des données recueillies, jusqu'à l'écriture de la recherche. De telles catégorisations en règle ou en norme figée ne rendent pas compte de processus dynamiques et situés. Comme l'évoquent plusieurs contributions, c'est dans le mouvement et l'instabilité que les chercheurs développent leurs pratiques d'analyse. De plus, l'interrelation du chercheur avec le phénomène qu'il étudie contribue à ne pas réifier des manières de faire qui pourraient limiter la compréhension d'un phénomène. Si certains auteurs abordent la question du rapport étic ou émic du chercheur et de l'objet, en évoquant les liens entre analyse d'un phénomène et le vécu du chercheur, d'autres auteurs, ont au contraire, une approche plus neutre à l'objet étudié, même si la dimension du chercheur en tant que sujet agissant est toujours perceptible dans la relation à son objet de recherche.

\section{Linvitation à poursuivre les investigations}

Généralement, les manières dont s'y prend un chercheur en SHS, en liaison avec différents contextes de production, ne sont que peu exposées, expliquées ou explicitées. Ces manières sont la face visible des conceptualisations qu'il développe, en interne, dans son activité de recherche. Le recueil de ce que les acteurs, œuvrant dans différents contextes, disent à propos de leurs vécus, apporte une pierre à ce que seraient, pour eux, des ficelles lorsqu'il s'agit de mener l'analyse d'un phénomène en SHS ou de répondre à des problèmes qui se posent au cours de leur activité. Et, même, si les exemples ne soulèvent qu'une partie des questionnements, ils contribuent déjà à rendre compte de la richesse de débats et d'échanges autour de questions vives qui traversent les démarches de recherche, quelle que soit l'approche épistémologique mobilisée. Le questionnement qui tourne autour de ce que pourraient être ces ficelles n'est pas l'apanage d'une ancienneté dans le métier de chercheur. II est aussi un processus continu tout au long de la vie du chercheur, de ses objets et de ses rencontres. Certaines contributions sont issues d'un travail réflexif conjoint de jeunes chercheurs avec leurs encadrants qui les amènent à faire des propositions sur le processus d'analyse des données, voire à s'interroger sur l'utilisation de résultats de recherche dans une perspective de données probantes. 
D'autres contributions reflètent le questionnement de chercheurs plus confirmés sur leur méthodologie ou sur l'agir avec les participants à la recherche, ou plus généralement sur comment leur cheminement de recherche les amène à questionner la formation des étudiants. L'échange croisé, la confrontation aux autres, et la réflexivité amènent à enrichir et à reconfigurer les schèmes pour son activité. L'interdisciplinarité tout particulièrement conduit à expérimenter de nouvelles manières de faire et de construire un savoir commun.

Les articles réunis constituent des traces de l'activité des chercheurs sur leur méthodologie: ils donnent à voir leurs pratiques de manière aussi descriptive que possible, pour que d'autres puissent s'en saisir afin de les discuter. Mettre en réflexivité les chercheurs, attachés à cette entreprise, rend compte de processus de conceptualisations qui ne sont que grossièrement identifiés dans la métaphore de ficelles, de bricolage. D'autres approches et méthodes, autres que centrées sur l'écrit, restent bien entendu possibles et à développer pour accéder à ces conceptualisations. De manière générale, la mise en mots de son activité, que cela soit à titre individuel ou de manière collaborative, sert d'objet intermédiaire pour développer la conduite de recherches en SHS.

La conceptualisation, selon une analyse fine, en relation avec des désirs, des intentions et des objectifs, et prenant en compte une élaboration critique de ses propres pratiques avec ses règles et ses inférences, contribue à faire émerger, à décomposer et à rendre visible, aussi bien pour le chercheur lui-même que pour d'autres, ce qui est en jeu dans l'activité. Les conceptualisations des méthodes présentées ici ont une dimension épistémologique en tant qu'elles construisent par elles-mêmes et en elles-mêmes la connaissance. Autrement dit, l'exercice de l'explicitation des méthodes, au travers des articles, quelle que soit la forme que cette explicitation prend, éclaire les rapports des scientifiques à ce qui fait science ou comment ils élaborent la science.

\section{Bibliographie:}

Allamel-Raffin, C., Dupouy, S. et Gangloff, J.-L. (2019). Introduction. Philosopher sur l'expérimentation scientifique: bilan et perspectives. Philosophia Scientiæ [En ligne], 23 (2). URL: http://journals.openedition.org/philosophiascientiae/1899

Becker, H. (2002). Les ficelles du métier, Comment conduire sa recherche en sciences sociales. Paris: La Découverte.

Belpaume, C. (2009). Accompagner l'apprentissage du raisonnement clinique en soins infirmiers. Recherche en soins infirmiers, 99 (4), 43-74.

Boch, F. et Rinck, F. (2010). Pour une approche énonciative de l'écrit scientifique. Lidil, 41, 5-14.

Capdevielle-Mougnibas, V. et de Léonardis, M. (2010). Ségrégation sociale et responsabilité du chercheur: donner la parole à ceux qui ne l'ont pas. Recherches qualitatives, 29 (2), 132-159.

Clanet, J. et Talbot, L. (2012). Analyse des pratiques d'enseignement: éléments de cadrages théoriques et méthodologiques. Phronesis, $1(3), 4-18$.

Clot, Y., Faïta, D., Fernandez, G. et Scheller, L. (2000). Entretiens en autoconfrontation croisée: une méthode en clinique de l'activité. Perspectives Interdisciplinaires Sur Le Travail et La Santé (PISTE), 2 (1).

Crettaz, B. (1986). Le chercheur en sciences humaines et son « métier »: artisan ou bricoleur? [en ligne] Cahiers de I'IUED, 143-162.

Daoust, M. (2018). Sciences normatives, procédures neutres. Philosophia Scientiæ, 22 (2), 37-57.

Deschenaux, F. (2013). Introduction Des bonnes pratiques à diffuser comme adjuvant à la reconnaissance de la recherche qualitative. Recherches qualitatives, 32 (1), 1-6.

Desgagné, S., (1997). Le concept de recherche collaborative: I'idée d'un rapprochement entre chercheurs universitaires et praticiens enseignants. Revue des sciences de l'éducation, 23 (2), 371-393. 
Fassin, D. (2008). Extension du domaine de l'éthique. Mouvements, 55-56 (3), 124-127.

Fossion, G., Jamin, V. et Faulx, D. (2018). Investiguer les creux du discours par la technique d'entretien de la mise au carré. Recherches qualitatives, 37 (1), $21-56$.

Gingras, Y. (1991). L'institutionnalisation de la recherche en milieu universitaire et ses effets. Sociologie et sociétés, 23 (1), 41-54.

Gremion, L. (2016). « Tu sais, tu ne nous apprends rien! » Ethnographie des coulisses professionnelles de la discrimination scolaire. Dans J.-P. Payet. Ethnographie de l'école. Les coulisses des institutions scolaires et socio-éducatives, (pp.141-161). Rennes: Presses universitaires de Rennes.

Goigoux, R. (2007). Un modèle d'analyse de l'activité des enseignants. Éducation et didactique, 1 (3), 47-70.

Grossi, E.P. (2005). Uma arqueologia dos saberes do GEMMPA. Revista do GEMMPA. Todos podem aprender: qual é a chave?, 10, 11-40.

Hackett, E. J. (2005). Essential Tensions: Identity, Control, and Risk in Research. Social Studies of Science, 35 (5), 787-826.

Hacking, I. (2005). Les philosophes de l'expérience. Tracés, 9, 67-82, doi: 10.4000/traces.201, trad. fr de Philosophers of Experiment, PSA: Proceedings of the Biennial Meeting of the Philosophy of Science Association, 1988/2, 147-156.

Jouvenet, M. (2007). La culture du "bricolage" instrumental et l'organisation du travail scientifique: enquête dans un centre de recherche en nanosciences. Revue d'anthropologie des connaissances, 1 (2), 189-219.

Larouche, J. (2019). Les sciences sociales et l'éthique en recherche en contexte canadien: Régulation imposée ou approche réflexive? Revue d'anthropologie des connaissances, 2 (2), 479-501.

Latour, B. (2005). La science en action. Paris: La Découverte.

Latour, B. (2007). Changer de société: refaire de la sociologie. Paris: La Découverte.

Morissette, J., Pagoni, M. et Pépin, M. (2017). De la cohérence épistémologique de la posture collaborative. Phronesis, 6 (1-2), 1-7.

Niewiadomski, C., Portelance, L. et Perez-Roux., T. (2017). Introduction. Collaborations chercheur(s)-praticien(s): nouvelles formes, nouveaux enjeux? Éducation et socialisation [Online], 45 | 2017, Online since 01 September 2017, connection on 03 November 2020. URL: http://journals.openedition.org/edso/2482; DOI: https://doi.org/10.4000/edso.2482

Numa-Bocage L. (2014). De l'observation comme instrument psychologique pour le chercheur et l'enseignant. Revue Recherche en Éducation, 19, 40-51.

Paillé, P., Muchielli, A. (2003). L'analyse qualitative en sciences humaines et sociales. Paris: Armand Colin

Pastré, P. (1999). La conceptualisation dans l'action: bilan et nouvelles perspectives. Éducation permanente, 139, $13-36$.

Pastré, P. (2002). L'analyse du travail en didactique professionnelle. Revue française de pédagogie, 138, 9-17.

Pastré, P., Mayen, P. et Vergnaud, G. (2006). La didactique professionnelle. Revue Française de Pédagogie, 154, 145-198.

Rabardel, P. (2005). Instrument subjectif et développement du pouvoir d'agir. Dans P. Rabardel et P. Pastré (Dir.). Modèles du sujet pour la conception. Dialectiques, activités, développement, (pp. 11-29). Toulouse: Octarès.

Raynaud, D. (1999). Le schème: opérateur de la conception architecturale. Intellectica - La revue de l'Association pour la Recherche sur les sciences de la Cognition (ARCo), 29 (2), 35-69. 
Rix-Lièvre, G. (2010). Différents modes de confrontation à des traces de sa propre activité: Vers une confrontation à une perspective subjective située. Revue d'anthropologie des connaissances, 4 (2), 358-379.

Rogalski, J. (2004). La didactique professionnelle: une alternative aux approches de « cognition située » et « cognitiviste » en psychologie des acquisitions. @ctivités, 1 (2), 103-120.

Soler, L. et Zwart, S. D. (2013). Editorial Introduction: Collins and Tacit Knowledge. Philosophia Scientiæ, 17 (3), 5-23.

Spire, A. (1999). De l'étranger à l'immigré. Actes de la recherche en sciences sociales, 129, 50-56.

Van Campenhoudt, L. (2000). La responsabilité de la recherche universitaire. Éthique publique [En ligne], 2 (2). URL: http://journals.openedition.org/ethiquepublique/2669.

Vergnaud, G. et Récopé, M. (2000). De Revault d'Allonnes à une théorie du schème aujourd'hui. Psychologie française. 45 (1), 35-50.

Vergnaud, G. (1985). Concepts et schèmes dans une théorie opératoire de la représentation. Psychologie française, 30, 245-252

Vergnaud, G. (1996). Au fond de l'action la conceptualisation. Dans J.-M. Barbier (Dir.), Savoirs théoriques et savoirs d'action (pp. 275292). Paris: Presses universitaires de France.

Vinck, D. (2009). De l'objet intermédiaire à l'objet frontière. Revue d'anthropologie des connaissances, 3 (1), 51-72. 